\title{
Ba with Unusual Oxidation States in Ba Chalcogenides under Pressure
}

Fei $\mathrm{Li}^{1,2 \dagger}$, Xiaohua Zhang ${ }^{1,2 \dagger}$,Yang Fu ${ }^{1}$, Yanchao Wang ${ }^{3, *}$, Aitor Bergara ${ }^{4,5,6,{ }^{*}}$ and Guochun Yang ${ }^{1,2 *}$

${ }^{I}$ State Key Laboratory of Metastable Materials Science \& Technology and Key Laboratory for Microstructural Material Physics of Hebei Province, School of Science, Yanshan University, Qinhuangdao 066004, China

${ }^{2}$ Centre for Advanced Optoelectronic Functional Materials Research and Key Laboratory for UV Light-Emitting Materials and Technology of Ministry of Education, Northeast Normal University, Changchun 130024, China

${ }^{3}$ International Center of Computational Method and Software, State Key Laboratory of Superhard Materials, College of Physics, Jilin University, Changchun 130012, China

${ }^{4}$ Departamento de Física de la Materia Condensada, Universidad del País Vasco-Euskal Herriko Unibertsitatea, UPV/EHU, 48080 Bilbao, Spain

${ }^{5}$ Donostia International Physics Center (DIPC), 20018 Donostia, Spain

${ }^{6}$ Centro de Física de Materiales CFM, Centro Mixto CSIC-UPV/EHU, 20018 Donostia, Spain

Index

Page

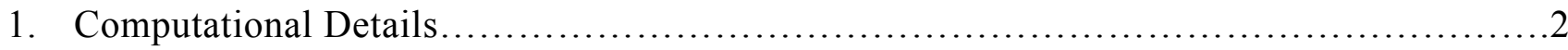

2. Calculated Birch-Murnaghan equation of states for $F m-3 m$ BaS ........................ 4

3. Phase stability of various $\mathrm{Ba}-\mathrm{Ch}$ compounds..........................................

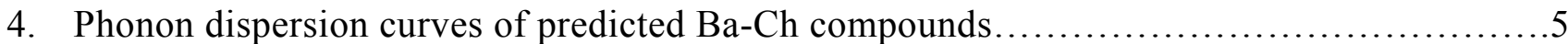

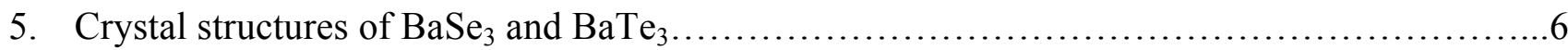

6. Energy band structures and projected density of states of various Ba-Ch compounds............7

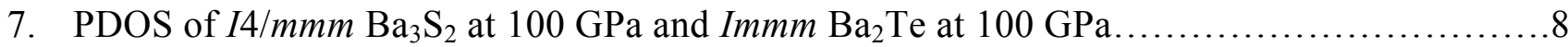

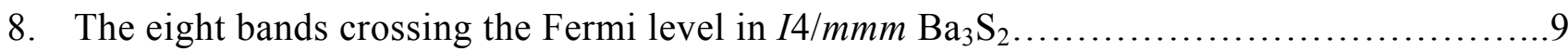

9. The calculated Löwdin and Mulliken charge of $\mathrm{Ba}(1)$ and $\mathrm{Ba}(2)$ in $I 4 / m m m ~ \mathrm{Ba}_{3} \mathrm{~S}_{2} \ldots \ldots \ldots 10$

10. The calculated Löwdin and Mulliken charge of Ba in Im $m$ $\begin{array}{llllllllllllllllllll}\mathrm{B} & \mathrm{a} & 2 & \mathrm{~T} & \mathrm{e} & \ldots & \ldots & \ldots & \ldots & \ldots & \ldots & \ldots & \ldots & \ldots & \ldots & . & \ldots & 1 & 0\end{array}$

11. Structural parameters of the predicted stable Ba-Ch compounds.......................... 10

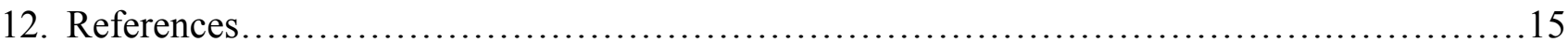




\section{Computational Details}

Our structure prediction approach is based on a global minimization of free energy surfaces merging ab initio total-energy calculations as implemented in the CALYPSO code ${ }^{1,2}$.Searches were carried out to identify the most stable structures with stoichiometries of $\mathrm{Ba}_{x} \mathrm{Ch}_{y}(x=1, y=1-4$ or $x=$ 2, $y=1$ and 3 , or $x=3, y=2$ ) using simulation cell sizes of 1-4 formula units (f.u.) at $0 \mathrm{~K}$ and the considered pressures of 1 atm, 25, 50, 100, and $150 \mathrm{GPa}$. In the first step, random symmetric structures are constructed in which atomic coordinates are generated by the crystallographic symmetry operations. Local optimizations using the VASP $\operatorname{code}^{3}$ are done with the conjugate gradient method, and are deemed to be converged when the enthalpy changes become smaller than 1 $\times 10^{-5} \mathrm{eV}$ per cell. After the first generation of structures is optimized $60 \%$ of the lowest lying structures are selected to construct the next generation by PSO (Particle Swarm Optimization). 40\% of the structures in the new generation are randomly generated. A structure fingerprinting technique using a bond characterization matrix is applied to the generated structures, so that identical structures are strictly forbidden. This procedure significantly enhances the diversity of the structures, which is crucial for maintaining the efficiency of the global search. In most cases, structure search simulations for each calculation are stopped after generating $1000 \sim 1200$ structures (e.g., about $20 \sim 30$ generations).

To further analyze the structures with higher accuracy, we select a number of structures with lower total enthalpies and perform structural optimization using density functional theory within the generalized gradient approximation (GGA) $)^{4}$ as implemented in the VASP code. The cut-off energy for the expansion of wavefunctions into plane waves is set to $500 \mathrm{eV}$ in all calculations, and a Monkhorst-Pack $k$-mesh with a maximum spacing of $2 \pi \times 0.03 \AA^{-1}$. This usually gives enthalpies well converged to within $\sim 1 \mathrm{meV} /$ atom. The electron-ion interaction is described by pseudopotentials built within the scalar relativistic projector augmented-wave (PAW) method with $5 \mathrm{~s}^{2} 5 \mathrm{p}^{6} 6 \mathrm{~s}^{2}$ and $n \mathrm{~s}^{2} n \mathrm{p}^{4}(n=2,3,4$ and 5) valence electrons for Ba and Ch atoms, respectively.

To determine the dynamic stability of the predicted structures, phonon calculations are performed using the finite displacement approach ${ }^{5}$ as implemented in the Phonopy $\operatorname{code}^{6}$. In order to further test the reliability of the adopted pseudopotentials for $\mathrm{Ba}$ and $\mathrm{S}$, the validity of the projector augmented wave pseudopotentials from the VASP library is checked by comparing the calculated 
Birch-Murnaghan equation of state of CsCl-type BaS with that obtained with the full-potential linearized augmented plane-wave method (LAPW) using local orbitals (as implemented in WIEN2k) ${ }^{7}$. The Birch-Murnaghan equation of states derived from PAW and LAPW methods are almost identical (Figure S1). Thus, our adopted pseudopotentials are feasible in the range of $0 \sim 300$ GPa. In addition, spin polarization calculations were also performed. All of the Ba-Ch systems were found to be non-magnetic.

The electron-phonon coupling calculations are carried out with the density functional perturbation (linear response) theory as implemented in the QUANTUM ESPRESSO package ${ }^{8}$. We employ ultrasoft pseudopotentials with $5 s^{2} 5 p^{6} 6 s^{2}$, and $3 s^{2} 3 p^{4}$ as valence electrons for $\mathrm{Ba}$, and $\mathrm{S}$ atoms, respectively. The kinetic energy cutoff for wave-function expansion is chosen as $60 \mathrm{Ry}$. To reliably calculate electron-phonon coupling in metallic systems, we need to sample dense $k$-meshes for the electronic Brillouin zone integration and enough $q$-points for evaluating the average contributions from the phonon modes: We have used a $8 \times 8 \times 6 \mathrm{k}$-mesh and $4 \times 4 \times 3 \mathrm{q}$-mesh for calculating the superconducting $T_{\mathrm{c}}$. The EPC parameter $\lambda$ is calculated by using a set of Gaussian broadenings $(\sigma)$ in steps of 0.005 Ry from 0.005 to 0.050 Ry. The $8 \times 8 \times 6 \mathrm{k}$-grid appears to be converged for $\sigma=0.04$ Ry. We have calculated the superconducting $T_{\mathrm{c}}$ of $P 4_{1} 2_{1} 2$ phase $\mathrm{BaS}_{2}$ and $I 4 / \mathrm{mmm} \mathrm{Ba}_{3} \mathrm{~S}_{2}$ as estimated from the McMillan-Allen-Dynes formula ${ }^{9-11}$ :

$$
T_{\mathrm{c}}=\frac{\omega_{\log }}{1.2} \exp \left[-\frac{1.04(1+\lambda)}{\lambda-\mu^{*}(1+0.62 \lambda)}\right] .
$$

Here, $\mu^{*}$ is the Coulomb pseudopotential $\left(\mu^{*}=0.1\right)$. The electron-phonon coupling constant, $\lambda$, and the logarithmic average phonon frequency, $\omega_{\log }$, are calculated from the Eliashberg spectral function for the electron-phonon interaction:

$$
\alpha^{2} F(\omega)=\frac{1}{N\left(E_{F}\right)} \sum_{k q, v}\left|g_{k, k+q, v}\right|^{2} \delta\left(\varepsilon_{k}\right) \delta\left(\varepsilon_{k+q}\right) \delta\left(\omega-\omega_{q, v}\right)
$$

where $\lambda=2 \int d \omega \frac{\alpha^{2} F(\omega)}{\omega} ; \quad \omega_{\log }=\exp \left[\frac{2}{\lambda} \int \frac{d \omega}{\omega} \alpha^{2} F(\omega) \ln (\omega)\right]$. Herein, $N\left(E_{F}\right)$ is the electronic density of states at the Fermi level, $\omega_{q, v}$ is the phonon frequency of mode $v$ and wave vector $q$, and $\left|g_{k, k+q, v}\right|$ is the electron-phonon matrix element between two electronic states with momenta $k$ and $k+q$ at the Fermi level ${ }^{12,13}$. 


\section{Supporting Figures}

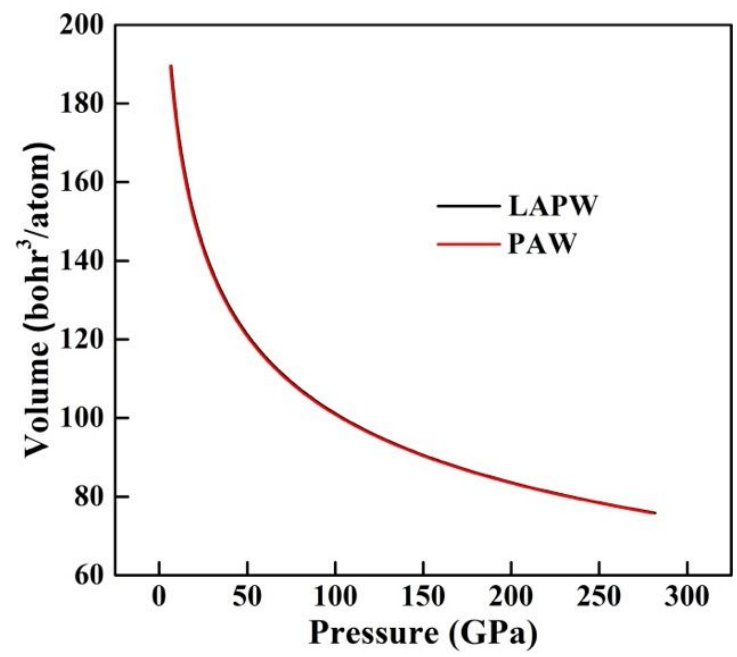

Figure S1. Comparison of the fitted Birch-Murnaghan equation of states for BaS with $F m-3 m$ symmetry by using the calculated results from the PAW pseudopotentials and the full-potential LAPW methods.
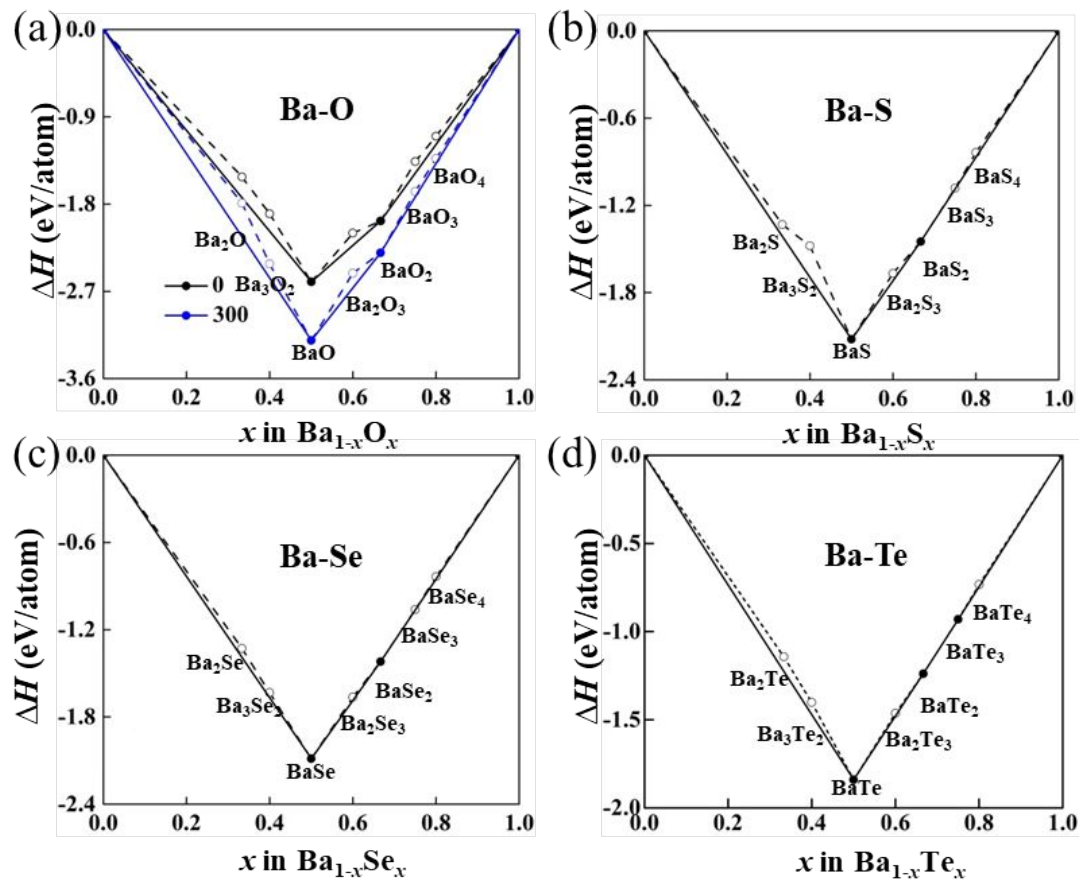

Figure S2. Phase stability of various Ba-Ch compounds for (a) Ba-O system (0 and $300 \mathrm{GPa})$, (b) Ba-S system (0 GPa), (c) Ba-Se system $(0 \mathrm{GPa})$ and (d) Ba-Te system (0 GPa). To further explore the potential stability of $\mathrm{Ba}_{3} \mathrm{O}_{2}$, we calculated the Convex hull of $\mathrm{Ba}-\mathrm{O}$ system at $300 \mathrm{GPa}$. The results show that the $\mathrm{Ba}_{3} \mathrm{O}_{2}$ is thermodynamically unstable even up to $300 \mathrm{GPa}$. 

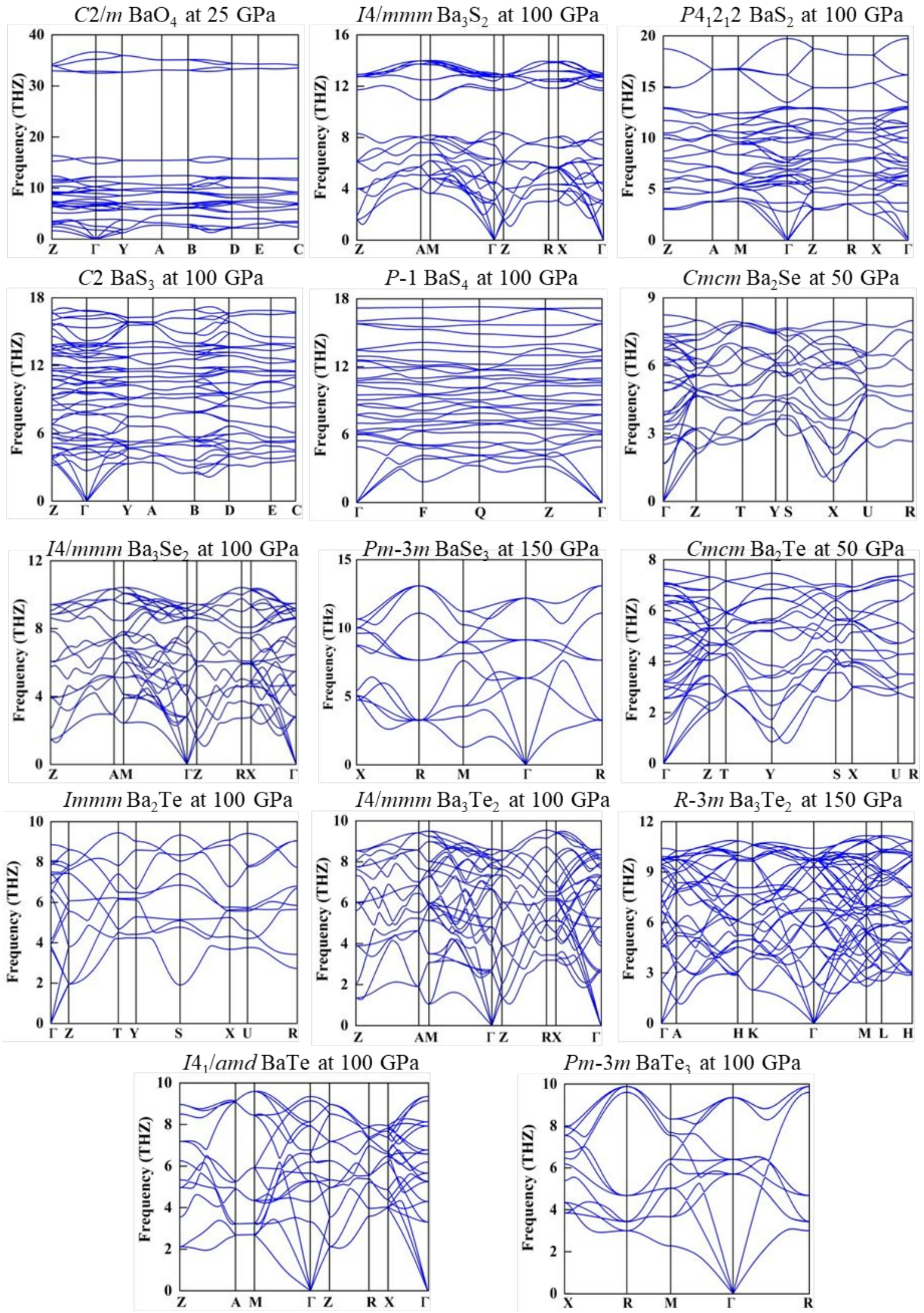

Figure S3. Phonon dispersion curves of the predicted Ba-Ch compounds. 


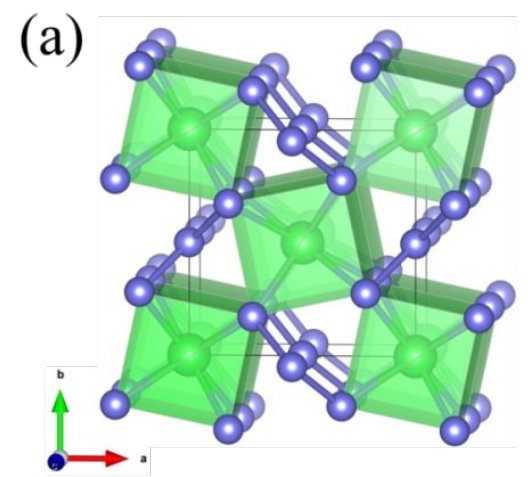

(c)

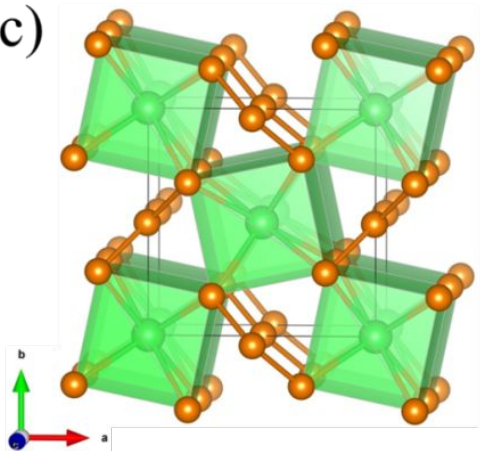

(b)

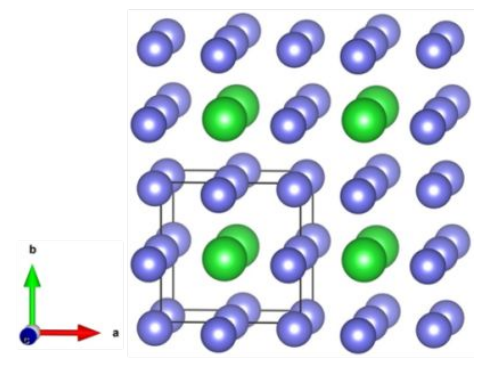

(d)

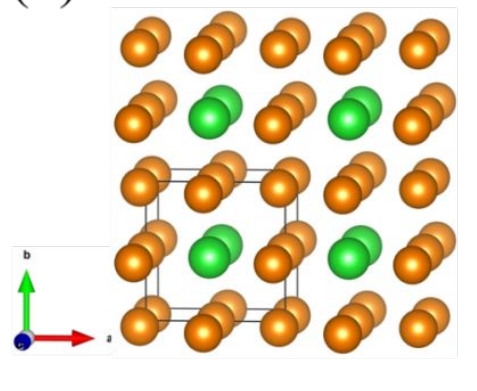

Figure S4. The crystal structures identified by our structural prediction. (a) $P-42_{1} m \mathrm{BaSe}_{3}$, (b) $P m-3 m \mathrm{BaSe}_{3}$, (c) $P-42_{1} m \mathrm{BaTe}_{3}$, and (d) $P m-3 m \mathrm{BaTe}_{3}$. In all these structures, green, blue, and orange spheres represent $\mathrm{Ba}, \mathrm{Se}$, and Te atoms, respectively. 

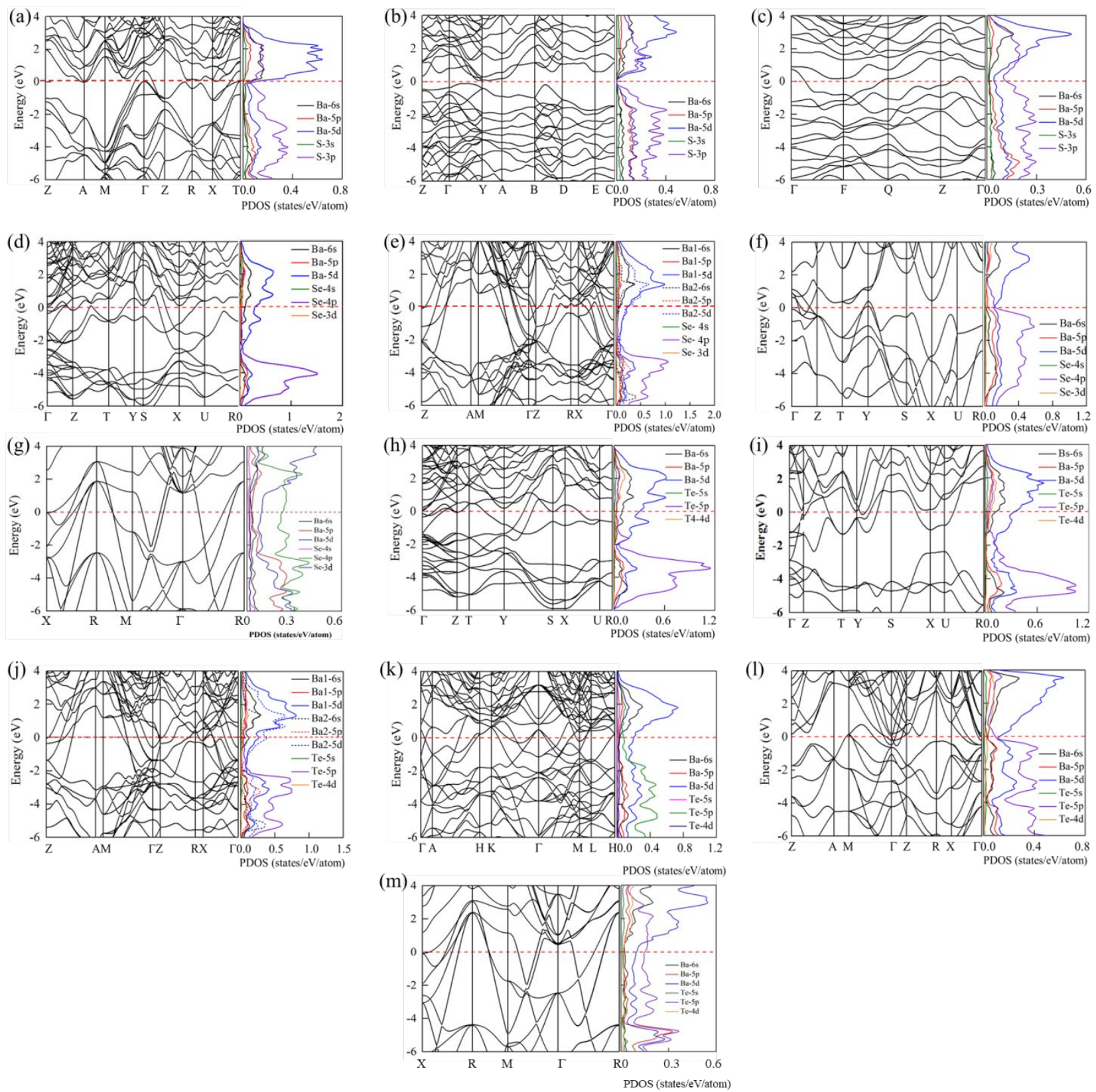

Figure S5. Energy band structures and projected density of states of (a) $P 4_{1} 2{ }_{1} 2 \mathrm{BaS}_{2}$ at $100 \mathrm{GPa}$, (b) $C 2 \mathrm{BaS}_{3}$ at $100 \mathrm{GPa}$, (c) $P-1 \mathrm{BaS}_{4}$ at $100 \mathrm{GPa}$, (d) $C m c m \mathrm{Ba}_{2} \mathrm{Se}$ at $50 \mathrm{GPa}$, (e) $I 4 / m m m \mathrm{Ba}_{3} \mathrm{Se}_{2}$ at $100 \mathrm{GPa}$, (f) Pmma BaSe at $150 \mathrm{GPa}$, (g) Pm-3m BaSe 3 at $150 \mathrm{GPa}$, (h) Cmcm $\mathrm{Ba}_{2} \mathrm{Te}$ at $50 \mathrm{GPa}$, (i) Immm $\mathrm{Ba}_{2} \mathrm{Te}$ at $100 \mathrm{GPa}$, (j) I4/mmm $\mathrm{Ba}_{3} \mathrm{Te}_{2}$ at $100 \mathrm{GPa}$, (k) R-3m $\mathrm{Ba}_{3} \mathrm{Te}_{2}$ at $150 \mathrm{GPa}$, (l) I4 ${ }_{1} /$ amd BaTe at $150 \mathrm{GPa}$, and (m) Pm-3m $\mathrm{BaTe}_{3}$ at $100 \mathrm{GPa}$. 

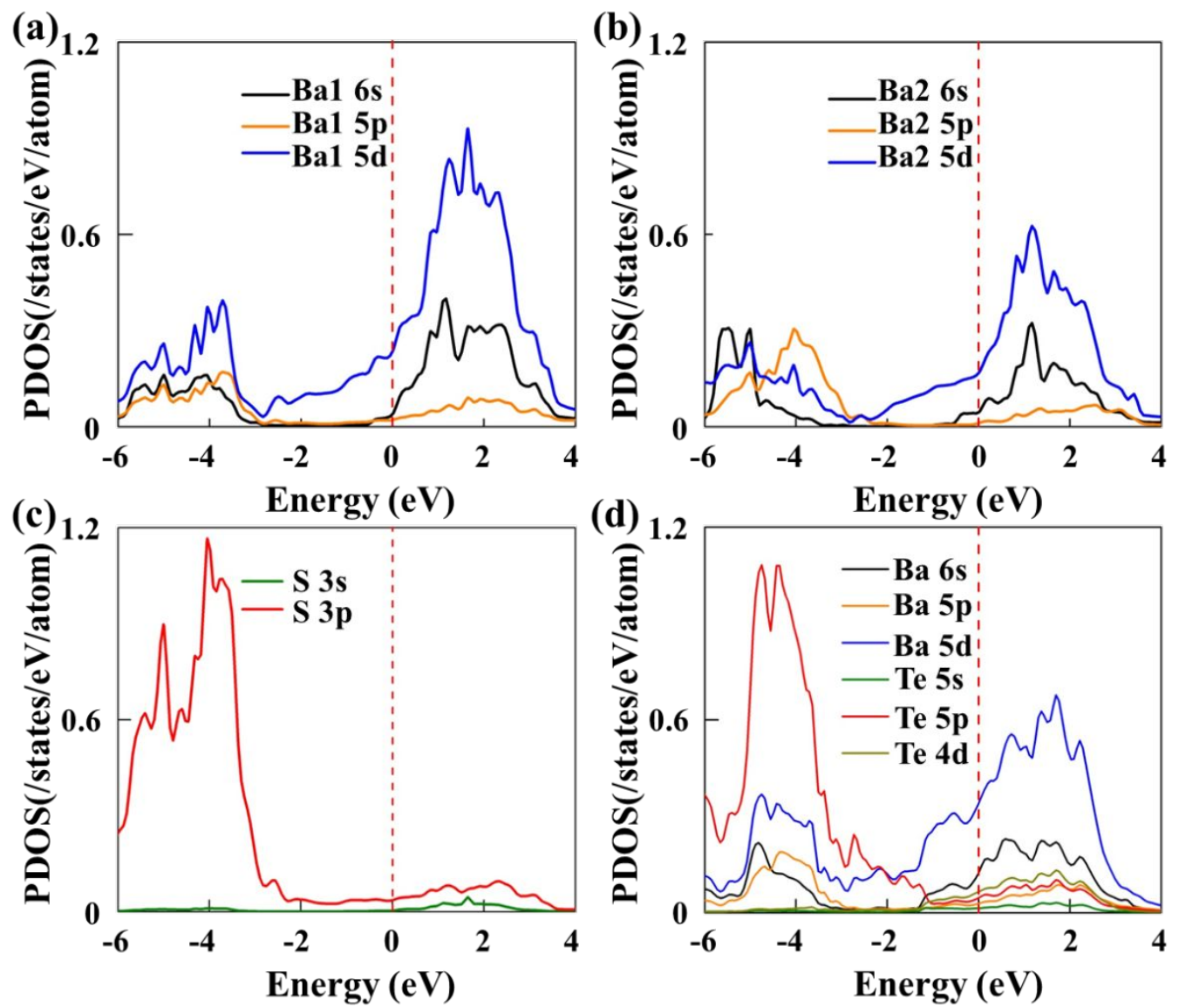

Figure S6. PDOS of $I 4 / \mathrm{mmm} \mathrm{Ba}_{3} \mathrm{~S}_{2}$ at $100 \mathrm{GPa}$ and $I m m m \mathrm{Ba}_{2} \mathrm{Te}$ at $100 \mathrm{GPa}$. Considering that there exist two types of $\mathrm{Ba}$ atoms with different valence states in $\mathrm{Ba}_{3} \mathrm{~S}_{2}$, the PODS of $I 4 / m m m \mathrm{Ba}_{3} \mathrm{~S}_{2}$ contributed by $\mathrm{Ba} 1, \mathrm{Ba} 2$ and $\mathrm{S}$ are shown in (a), (b), and (c) respectively. The PDOS of Immm $\mathrm{Ba}_{2} \mathrm{Te}$ is shown in (d). 

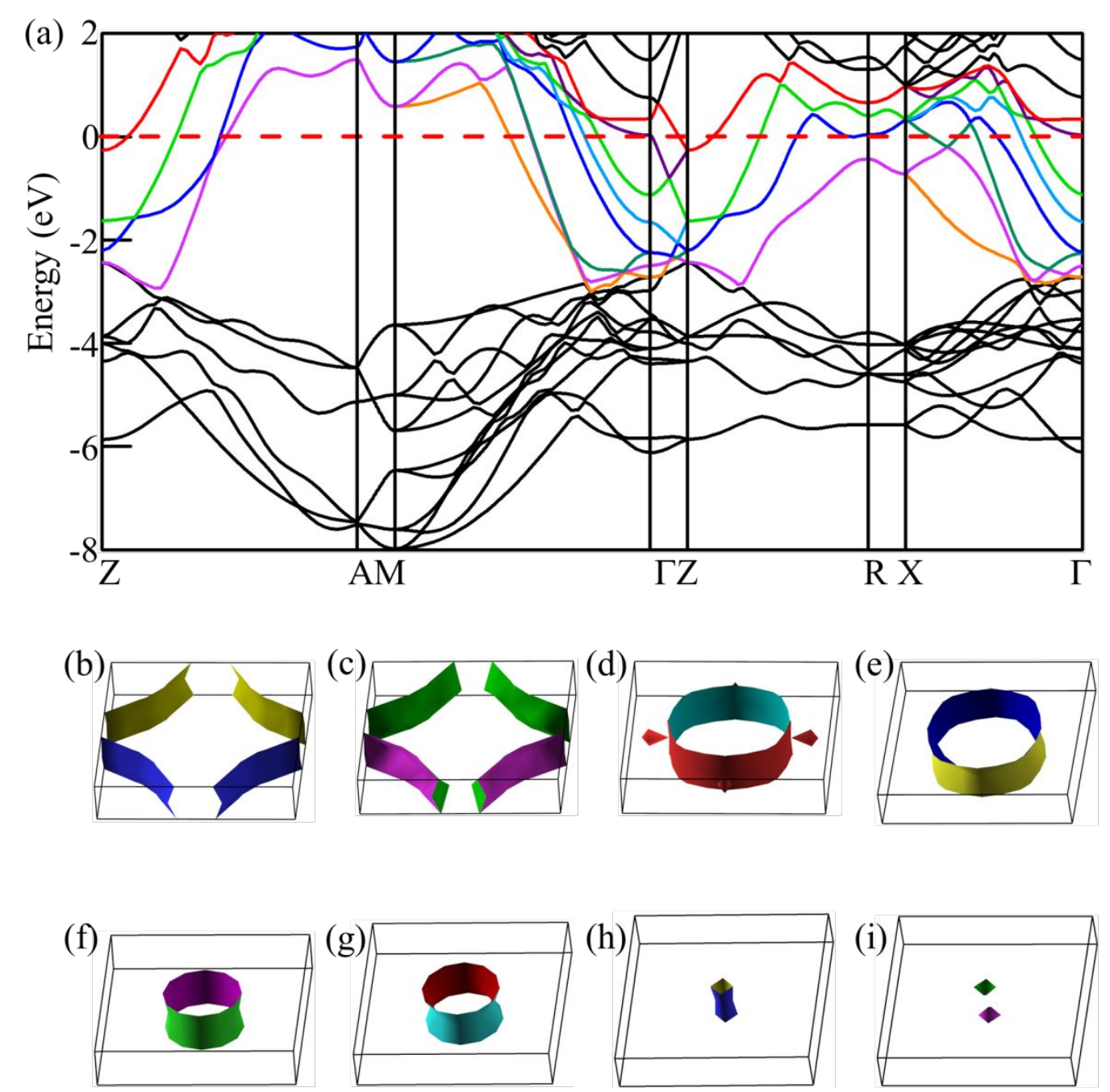

Figure S7. (a) The eight bands crossing the Fermi level in $I 4 / \mathrm{mmm} \mathrm{Ba}_{3} \mathrm{~S}_{2}$ at $100 \mathrm{GPa}$. (b), (c), (d), (e), (f), (g), (h), and (i) represent the Fermi surfaces corresponding to orange, light purple, dark green, blue, light blue, light green, purple and red bands in (a). It is noting that the Fermi surfaces of (b), (c), (d), (e), (f), and (g) mainly contribute to the electrical conductivity of the $I 4 / \mathrm{mmm} \mathrm{Ba}_{3} \mathrm{~S}_{2}$ in $a b$ plane, whereas the Fermi surfaces of (h) and (i) negligibly contribute to the electrical conductivity of the $I 4 / \mathrm{mmm} \mathrm{Ba}_{3} \mathrm{~S}_{2}$ in $c$ axis. 
Table S1. The calculated Löwdin and Mulliken charge of $\mathrm{Ba}(1)$ and $\mathrm{Ba}(2)$ in $I 4 / m m m ~ \mathrm{Ba}_{3} \mathrm{~S}_{2}$.

\begin{tabular}{ccc}
\hline \hline Atom & Löwdin & Mulliken \\
\hline $\mathrm{Ba}(1)$ & 0.80 & 0.83 \\
$\mathrm{Ba}(2)$ & 1.48 & 1.46 \\
$\mathrm{~S}$ & -1.54 & -1.56 \\
\hline
\end{tabular}

Table S2. The calculated Löwdin and Mulliken charge of Ba in Immm $\mathrm{Ba}_{2} \mathrm{Te}$.

\begin{tabular}{lll}
\hline Atom & Löwdin & Mulliken \\
\hline $\mathrm{Ba}$ & 0.99 & 1.02 \\
$\mathrm{Te}$ & -1.94 & -2.02 \\
\hline
\end{tabular}

Table S3. Structural parameters of the predicted stable Ba-Ch compounds

\begin{tabular}{|c|c|c|c|c|c|c|}
\hline \multirow[t]{2}{*}{ Phases } & \multirow{2}{*}{$\begin{array}{c}\text { Press } \\
\text { ure } \\
\text { (GPa) }\end{array}$} & \multirow{2}{*}{$\begin{array}{l}\text { Lattice } \\
\text { Parameters } \\
\left(\AA,{ }^{\circ}\right)\end{array}$} & \multirow[t]{2}{*}{ Atoms } & \multicolumn{3}{|c|}{$\begin{array}{c}\text { Wyckoff Positions } \\
\text { (fractional) }\end{array}$} \\
\hline & & & & $x$ & $y$ & $z$ \\
\hline \multirow[t]{6}{*}{$P m-3 m$ BaO } & 150 & $a=2.7747$ & $\mathrm{Ba}(1 \mathrm{a})$ & 0.0000 & 0.0000 & 0.0000 \\
\hline & & $b=2.7747$ & $\mathrm{O}(1 \mathrm{~b})$ & 0.5000 & 0.5000 & 0.5000 \\
\hline & & $c=2.7747$ & & & & \\
\hline & & $\alpha=90.0000$ & & & & \\
\hline & & $\beta=90.0000$ & & & & \\
\hline & & $\gamma=90.0000$ & & & & \\
\hline \multirow[t]{6}{*}{$C 2 / m \mathrm{BaO}_{2}$} & 150 & $a=6.3848$ & $\mathrm{Ba}(4 \mathrm{i})$ & 0.7200 & 0.000 & 0.9611 \\
\hline & & $b=5.6949$ & $\mathrm{O}(8 \mathrm{j})$ & 0.9357 & 0.7874 & 0.6309 \\
\hline & & $c=3.1938$ & & & & \\
\hline & & $\alpha=90.0000$ & & & & \\
\hline & & $\beta=112.1560$ & & & & \\
\hline & & $\gamma=90.0000$ & & & & \\
\hline$C 2 / m \mathrm{BaO}_{4}$ & 25 & $a=4.6915$ & $\mathrm{Ba}(2 \mathrm{~d})$ & 0.0000 & 0.5000 & 0.500 \\
\hline
\end{tabular}




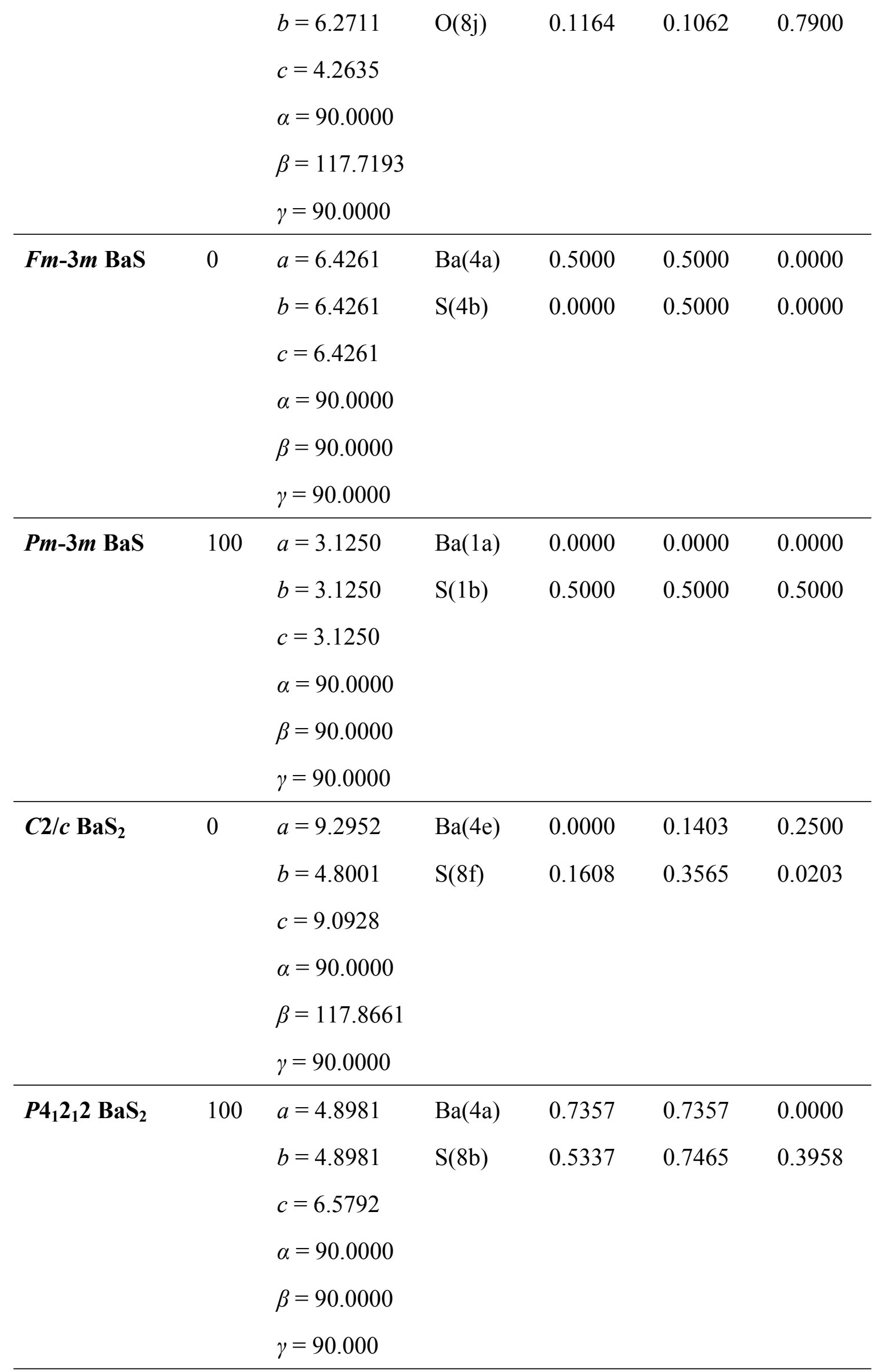




\begin{tabular}{|c|c|c|c|c|c|c|}
\hline \multirow[t]{6}{*}{$P-42_{1} m \mathrm{BaS}_{3}$} & 0 & $a=6.9509$ & $\mathrm{Ba}(2 \mathrm{a})$ & 0.0000 & 0.0000 & 0.0000 \\
\hline & & $b=6.9509$ & $\mathrm{~S}(2 \mathrm{c})$ & 0.5000 & 0.0000 & 0.7896 \\
\hline & & $c=4.2054$ & $\mathrm{~S}(4 \mathrm{e})$ & 0.3210 & 0.1790 & 0.5236 \\
\hline & & $\alpha=90.0000$ & & & & \\
\hline & & $\beta=90.0000$ & & & & \\
\hline & & $\gamma=90.0000$ & & & & \\
\hline \multirow[t]{6}{*}{$C 2 \mathrm{BaS}_{3}$} & 100 & $a=7.9301$ & $\mathrm{Ba}(2 \mathrm{a})$ & 0.0000 & 0.6624 & 0.0000 \\
\hline & & $b=4.6532$ & $\mathrm{Ba}(2 \mathrm{~b})$ & 0.0000 & 0.4416 & 0.5000 \\
\hline & & $c=5.6990$ & $S(4 c)$ & 0.8559 & 0.1620 & 0.0948 \\
\hline & & $\alpha=90.0000$ & $S(4 c)$ & 0.1455 & 0.9497 & 0.3892 \\
\hline & & $\beta=102.0970$ & $S(4 c)$ & 0.7646 & 0.8368 & 0.2676 \\
\hline & & $\gamma=90.0000$ & & & & \\
\hline \multirow[t]{6}{*}{$P-1 \mathrm{BaS}_{4}$} & 100 & $a=4.6355$ & $\mathrm{Ba}(2 \mathrm{i})$ & 0.7974 & 0.4555 & 0.1749 \\
\hline & & $b=4.7411$ & $S(2 i)$ & 0.6694 & 0.8538 & 0.7199 \\
\hline & & $c=5.7197$ & $\mathrm{~S}(2 \mathrm{i})$ & 0.6426 & 0.3510 & 0.6265 \\
\hline & & $\alpha=78.6483$ & $\mathrm{~S}(2 \mathrm{i})$ & 0.8382 & 0.8852 & 0.4197 \\
\hline & & $\beta=102.6659$ & $S(1 d)$ & 0.5000 & 0.0000 & 0.0000 \\
\hline & & $\gamma=90.7405$ & $\mathrm{~S}(1 \mathrm{a})$ & 0.0000 & 0.0000 & 0.0000 \\
\hline \multirow[t]{6}{*}{$I 4 / m m m \mathrm{Ba}_{3} \mathrm{~S}_{2}$} & 100 & $a=3.1807$ & $\mathrm{Ba}(2 \mathrm{a})$ & 0.0000 & 0.0000 & 0.0000 \\
\hline & & $b=3.1807$ & $\mathrm{Ba}(4 \mathrm{e})$ & 0.5000 & 0.5000 & 0.3068 \\
\hline & & $c=15.2616$ & $\mathrm{~S}(4 \mathrm{e})$ & 0.5000 & 0.5000 & 0.8992 \\
\hline & & $\alpha=90.0000$ & & & & \\
\hline & & $\beta=90.0000$ & & & & \\
\hline & & $\gamma=90.0000$ & & & & \\
\hline \multirow[t]{5}{*}{ Pmma BaSe } & 150 & $a=4.4170$ & $\mathrm{Ba}(2 \mathrm{e})$ & 0.2500 & 0.0000 & -0.7937 \\
\hline & & $b=2.9507$ & $\operatorname{Se}(2 f)$ & 0.2500 & 0.5000 & -0.2927 \\
\hline & & $c=4.4626$ & & & & \\
\hline & & $\alpha=90.0000$ & & & & \\
\hline & & $\beta=90.0000$ & & & & \\
\hline
\end{tabular}


$\gamma=90.0000$

\begin{tabular}{|c|c|c|c|c|c|c|}
\hline \multirow[t]{6}{*}{ Cmcm $\mathrm{Ba}_{2} \mathrm{Se}$} & 50 & $a=3.5005$ & $\mathrm{Ba}(4 \mathrm{c})$ & 0.5000 & 0.2634 & 0.7500 \\
\hline & & $b=13.8826$ & $\mathrm{Ba}(4 \mathrm{c})$ & 0.5000 & 0.9298 & 0.7500 \\
\hline & & $c=4.9444$ & $\operatorname{Se}(4 c)$ & 0.0000 & 0.0971 & 0.7500 \\
\hline & & $\alpha=90.0000$ & & & & \\
\hline & & $\beta=90.0000$ & & & & \\
\hline & & $\gamma=90.0000$ & & & & \\
\hline \multirow[t]{6}{*}{$I 4 / m m m \mathrm{Ba}_{3} \mathrm{Se}_{2}$} & 100 & $a=3.2849$ & $\mathrm{Ba}(2 \mathrm{a})$ & 0.0000 & 0.0000 & 0.0000 \\
\hline & & $b=3.2849$ & $\mathrm{Ba}(4 \mathrm{e})$ & 0.5000 & 0.5000 & 0.3028 \\
\hline & & $c=15.3049$ & $\operatorname{Se}(4 e)$ & 0.5000 & 0.5000 & 0.8982 \\
\hline & & $\alpha=90.0000$ & & & & \\
\hline & & $\beta=90.0000$ & & & & \\
\hline & & $\gamma=90.0000$ & & & & \\
\hline \multirow[t]{6}{*}{$P m-3 m \mathrm{BaSe}_{3}$} & 150 & $a=3.7254$ & $\mathrm{Ba}(1 \mathrm{~b})$ & 0.5000 & 0.5000 & 0.5000 \\
\hline & & $b=3.7254$ & $\operatorname{Se}(3 d)$ & 0.0000 & 0.0000 & 0.5000 \\
\hline & & $c=3.7254$ & & & & \\
\hline & & $\alpha=90.0000$ & & & & \\
\hline & & $\beta=90.0000$ & & & & \\
\hline & & $\gamma=90.0000$ & & & & \\
\hline \multirow[t]{6}{*}{ Cmcm $\mathrm{Ba}_{2} \mathrm{Te}$} & 50 & $a=3.6073$ & $\mathrm{Ba}(4 \mathrm{c})$ & -0.5000 & 0.7329 & 0.2500 \\
\hline & & $b=14.3561$ & $\mathrm{Ba}(4 \mathrm{c})$ & -0.5000 & 0.9270 & 0.7500 \\
\hline & & $c=5.0621$ & $\operatorname{Te}(4 c)$ & 0.0000 & 0.0970 & 0.7500 \\
\hline & & $\alpha=90.0000$ & & & & \\
\hline & & $\beta=90.0000$ & & & & \\
\hline & & $\gamma=90.0000$ & & & & \\
\hline \multirow[t]{4}{*}{ Immm Ba $\mathrm{Ba}_{2} \mathrm{Te}$} & 100 & $a=3.2433$ & $\mathrm{Ba}(4 \mathrm{i})$ & 0.0000 & 0.0000 & 0.1697 \\
\hline & & $b=3.6885$ & $\operatorname{Te}(2 \mathrm{c})$ & 0.5000 & 0.5000 & 0.0000 \\
\hline & & $c=9.0588$ & & & & \\
\hline & & $\alpha=90.0000$ & & & & \\
\hline
\end{tabular}




\begin{tabular}{|c|c|c|c|c|c|c|}
\hline & & $\begin{array}{l}\beta=90.0000 \\
\gamma=90.0000\end{array}$ & & & & \\
\hline \multirow{7}{*}{$\begin{array}{l}I 4 / m m m \\
\mathrm{Ba}_{3} \mathrm{Te}_{2}\end{array}$} & 100 & $a=3.4449$ & $\mathrm{Ba}(2 \mathrm{a})$ & 0.0000 & 0.0000 & 0.0000 \\
\hline & & & & & & \\
\hline & & $b=3.4449$ & $\mathrm{Ba}(4 \mathrm{e})$ & 0.5000 & 0.5000 & 0.2978 \\
\hline & & $c=15.3698$ & $\mathrm{Te}(4 \mathrm{e})$ & 0.0000 & 0.0000 & 0.3974 \\
\hline & & $\alpha=90.0000$ & & & & \\
\hline & & $\beta=90.0000$ & & & & \\
\hline & & $\gamma=90.0000$ & & & & \\
\hline \multirow[t]{6}{*}{$P m-3 m \mathrm{BaTe}_{3}$} & 100 & $a=4.1124$ & $\mathrm{Ba}(1 \mathrm{~b})$ & 0.5000 & 0.5000 & 0.5000 \\
\hline & & $b=4.1124$ & $\operatorname{Te}(3 d)$ & 0.0000 & 0.0000 & 0.5000 \\
\hline & & $c=4.1124$ & & & & \\
\hline & & $\alpha=90.0000$ & & & & \\
\hline & & $\beta=90.0000$ & & & & \\
\hline & & $\gamma=90.0000$ & & & & \\
\hline \multirow[t]{6}{*}{ I4 $1_{1}$ amd BaTe } & 100 & $a=4.1319$ & $\mathrm{Ba}(4 \mathrm{a})$ & 1.0000 & 0.5000 & 0.2500 \\
\hline & & $b=4.1319$ & $\mathrm{Te}(4 \mathrm{~b})$ & 0.5000 & 0.0000 & 0.2500 \\
\hline & & $c=8.5223$ & & & & \\
\hline & & $\alpha=90.0000$ & & & & \\
\hline & & $\beta=90.0000$ & & & & \\
\hline & & $\gamma=90.0000$ & & & & \\
\hline \multirow[t]{6}{*}{$R-3 m \mathrm{Ba}_{3} \mathrm{Te}_{2}$} & 150 & $a=4.5233$ & $\mathrm{Ba}(3 \mathrm{a})$ & 0.0000 & 0.0000 & 0.0000 \\
\hline & & $b=4.5233$ & $\mathrm{Ba}(6 \mathrm{c})$ & 0.0000 & 0.0000 & 0.8034 \\
\hline & & $c=13.6169$ & $\operatorname{Te}(6 \mathrm{c})$ & 0.0000 & 0.0000 & 0.6000 \\
\hline & & $\alpha=90.0000$ & & & & \\
\hline & & $\beta=90.0000$ & & & & \\
\hline & & $\gamma=120.0000$ & & & & \\
\hline
\end{tabular}




\section{References:}

(1) Wang, Y.; Lv, J.; Zhu, L.; Ma, Y. Crystal Structure Prediction via Particle-Swarm Optimization. Phys. Rev. B 2010, 82, 94116.

(2) Wang, Y.; Lv, J.; Zhu, L.; Ma, Y. CALYPSO: A Method for Crystal Structure Prediction. Comput. Phys. Commun. 2012, 183, 2063-2070.

(3) Kresse, G.; Furthmüller, J. Efficient Iterative Schemes for Ab Initio Total-Energy Calculations Using a Plane-Wave Basis Set. Phys. Rev. B 1996, 54, 11169-11186.

(4) Perdew, J. P.; Chevary, J. A.; Vosko, S. H.; Jackson, K. A.; Pederson, M. R.; Singh, D. J.; Fiolhais, C. Atoms, Molecules, Solids, and Surfaces: Applications of the Generalized Gradient Approximation for Exchange and Correlation. Phys. Rev. B 1992, 46, 6671-6687.

(5) Parlinski, K.; Li, Z. Q.; Kawazoe, Y. First-Principles Determination of the Soft Mode in Cubic $\mathrm{ZrO}_{2}$. Phys. Rev. Lett. 1997, 78, 4063-4066.

(6) Togo, A.; Oba, F.; Tanaka, I. First-Principles Calculations of the Ferroelastic Transition between Rutile-Type and $\mathrm{CaCl}_{2}$-Type $\mathrm{SiO}_{2}$ at High Pressures. Phys. Rev. B 2008, 78, 134106.

(7) Blaha, P.; Schwarz, K.; Sorantin, P.; Trickey, S. B. Full-Potential, Linearized Augmented Plane Wave Programs for Crystalline Systems. Comput. Phys. Commun. 1990, 59, 399-415.

(8) Giannozzi, P.; Baroni, S.; Bonini, N.; Calandra, M.; Car, R.; Cavazzoni, C.; Ceresoli, D.; Chiarotti, G. L.; Cococcioni, M.; Dabo, I.; et al. QUANTUM ESPRESSO: A Modular and Open-Source Software Project for Quantum Simulations of Materials. J. Phys. Condens. Matter 2009, 21, 395502.

(9) Oliveira, L. N.; Gross, E. K. U.; Kohn, W. Density-Functional Theory for Superconductors. Phys. Rev. Lett. 1988, 60, 2430-2433.

(10) Lüders, M.; Marques, M. A. L.; Lathiotakis, N. N.; Floris, A.; Profeta, G.; Fast, L.; Continenza, A.; Massidda, S.; Gross, E. K. U. Ab Initio Theory of Superconductivity. I. Density Functional Formalism and Approximate Functionals. Phys. Rev. B 2005, 72, 24545.

(11) Marques, M. A. L.; Lüders, M.; Lathiotakis, N. N.; Profeta, G.; Floris, A.; Fast, L.; Continenza, A.; Gross, E. K. U.; Massidda, S. Ab Initio Theory of Superconductivity. II. Application to Elemental Metals. Phys. Rev. B 2005, 72, 24546.

(12) Allen, P. B.; Mitrović, B. Theory of Superconducting Tc. In Solid State Physics; Ehrenreich, H., Seitz, F., Turnbull, D. B. T.-S. S. P., Eds.; Academic Press, 1983; Vol. 37, pp 1-92.

(13) Carbotte, J. P. Properties of Boson-Exchange Superconductors. Rev. Mod. Phys. 1990, 62, $1027-1157$. 